\title{
Educación para el desarrollo sostenible: hacia una comunidad aprendiente, de investigación y de práctica ${ }^{2}$
}

\author{
Education for Sustainable Development: \\ Towards a learning community, on researching and practicing
}

\section{Resumen}

La educación para el desarrollo sostenible (EDS) posee unas perspectivas y plantea problemas específicos que llevan a pensar en cuál será la estrategia de acción más adecuada. Eso implica discernir los conceptos y principios de la acción educativa más propicios para implementar este tipo de educación a todo nivel. Este artículo de reflexión temática quiere contribuir a articular los conceptos pedagógicos y didácticos que tienen que ver con la EDS, lo que permite apreciar no solamente su pertinencia, sino también toda su complejidad.

Palabras clave: educación para el desarrollo sostenible, comunidad aprendiente, comunidad de práctica, comunidad de investigación, ética.

\begin{abstract}
Education for sustainable development (ESD) poses some views and specific problems that lead to think about what it would be the most appropriate strategy of action. It implies to discern the concepts and principles of educational action more suitable for this kid of high education level. This article aims to enhance the articulations of pedagogical and didactic concepts that deal with EDS, allowing to appreciate not only its relevance but also all its complexity.
\end{abstract}

Keywords: education for sustainable development, learner community, community of practice, research community, ethics.

Recibido: 26 de octubre de 2014, evaluado: 29 de octubre de 2014, aprobado: 19 de noviembre de 2014

1 Magíster en Ciencias Sociales, Políticas y Económicas, Universidad Católica de Paris - Institut d'études sociales (Francia). Licenciado en Teología, Pontificia Universidad Javeriana. Director de Investigaciones de la Sede Principal, Corporación Universitaria Minuto de Dios. Miembro de los Grupos de Investigación "Ciudadanía, Paz y Desarrollo" e "Innovaciones educativas y cambio social" (Uniminuto). Correo electrónico: cgjuliao@gmail.com

2 Artículo de reflexión teórica derivado de un proceso de investigación, que pretende conceptualizar y definir la perspectiva de la educación para el desarrollo sostenible (EDS) en el Proyecto Educativo de Uniminuto. 


\section{Un planteamiento introductorio}

\author{
La educación es el arma más poderosa \\ para cambiar el mundo.
}

Nelson Mandela

La complejidad del concepto de desarrollo sostenible (DS), ${ }^{3}$ como también la amplitud de las cuestiones que supone, lleva a preguntarse, en el marco de un proyecto educativo como el de la Corporación Universitaria Minuto de Dios (Uniminuto), sobre los medios pedagógicos y didácticos más adecuados para implementar una educación para el desarrollo sostenible (EDS). Esta complejidad, palpable en las discusiones de ciertos especialistas, invita a mostrarse prudentes y circunspectos al elegir una estrategia de acción pedagógica. No basta con algunas técnicas concretas y prácticas; primero, porque es claro que reducir lo complejo a lo simple es peor en casos de crisis; segundo, porque habría que preguntarse por qué esos gestos y no otros. Asimismo, persistir en clichés como "hay que", "no hay sino que", o en juicios morales del tipo "está bien" o "está mal", muestra los límites del discurso utilizado hasta ahora.

El Proyecto Educativo de Uniminuto plantea que, en el marco de la misión y la filosofía propia, la meta institucional es "formar para el desarrollo humano y social integral". En ese contexto, plantea que "compartimos un pensamiento social específico que nos lleva a buscar que todas las personas y comunidades, particularmente las menos favorecidas, logren un desarrollo integral" (Uniminuto, 2014, cap. 3). En consecuencia, el PEI es claro al afirmar que:

La comunidad universitaria de UNIMINUTO sabe que dicho pensamiento social específico está en permanente construcción mediante el diálogo con otras personas y comunidades académicas, la reflexión y la investigación incesante, y, sobre todo, las acciones prácticas de educación para el desarrollo en las que interviene y que incrementan la reflexión y el conocimiento en el marco de lo que se ha llamado "comunidades aprendientes". ${ }^{4}$ Por eso no pretende "sentar cátedra" con una u otra concepción definitiva del desarrollo, mucho más cuando "no se puede dar por sentado que el desarrollo sea un paradigma ya resuelto (...) ni que sea ineluctable asumir un determinado rumbo de desarrollo como si se tratara de una categoría teleológica definida por la vida misma" (Useche, O. 2012, p. 40). Lo que sí se puede hacer es dar otras miradas al desarrollo, en una perspectiva que asume la diversidad, la diferencia y la complejidad de la vida, y que propugna por "emprender los caminos del bio-desarrollo, 5 como búsqueda integral del buen vivir". (2014, p. 55)

A partir de dicho planteamiento institucional, se propone este artículo para contribuir a la reflexión sobre ¿cómo pensar en una EDS que integre las finalidades propias de la educación, a saber el desarrollo de la humanidad en las personas, entendida desde el principio de responsabilidad, no solo en sus relaciones con el mundo, sino, sobre todo, en sus relaciones con el otro, más allá de las fronteras geopolíticas y generacionales? Así, se

3 El desarrollo sostenible (DS) es el paradigma global de las Naciones Unidas. El concepto de DS fue descrito, en 1987, en el Informe de la Comisión de Bruntland, como un "desarrollo que satisface las necesidades de la generación presente, sin comprometer la capacidad de las generaciones futuras de satisfacer sus propias necesidades". La sostenibilidad sería así un paradigma para pensar y construir un futuro en donde las dimensiones ambientales, sociales, económicas y culturales estén equilibradas en la búsqueda de una mejor calidad de vida, es decir, de felicidad.

4 Las comunidades son organismos vivos cuyo soporte es un aprendizaje continuo. Organismo que no aprende, muere. Una "comunidad aprendiente" es la que constituye un campo de relaciones altamente significativas en donde se producen experiencias, como la del conocer, ligadas a la vida. Allí confluyen la ecología de las subjetividades (la transformación de cada uno) con la ecología social (las relaciones con los otros-otras) y con la ecología del ambiente natural.

5 Con el concepto bio-desarrollo se hace alusión a la opción de un desarrollo para la vida en donde la acción social no se define solo en términos de progreso material, sino que se hace compatible con la diversidad y la complejidad, características de todo lo viviente. 
afirma que la EDS pretende que toda persona adquiera los saberes, las competencias, las actitudes y los valores requeridos para construir juntos un futuro sostenible, en el marco de un proceso de constitución de ciudadanía y de democracia, desde la perspectiva de la conformación de comunidades aprendientes, de investigación y de práctica, que permitan a cada sujeto ser autor/actor de su propio desarrollo. Igualmente, se afirma que este proyecto es ambicioso, pero necesario, hoy más que nunca, si se quiere preservar la Vida misma.

Por eso, aquí se esbozará el problema de la articulación existente entre las cuestiones éticopolíticas y las acciones educativas, pedagógicas y didácticas que hay que promover en una "educación para el desarrollo", que, desde la perspectiva de Uniminuto, giran alrededor de los conceptos de responsabilidad, democracia, comunidad aprendiente, de investigación y de práctica. En ese sentido, este artículo de reflexión presenta unas aproximaciones teórico-prácticas a estos conceptos y deja unos interrogantes abiertos para la reflexión posterior: ¿cuáles son los conceptos y principios educativos que puedan ser soportes para la EDS?, ¿con cuáles corrientes pedagógicas y didácticas relacionarla?, ¿dónde se sitúa su validez y cómo apreciar su pertinencia?, ¿cuáles interpelaciones surgen?, ¿qué nos enseñan los obstáculos encontrados hasta ahora en las prácticas que hemos implementado, tanto en los programas académicos de Uniminuto como en el Centro de Educación para el Desarrollo (CED)? Tales interrogantes relacionan el nivel macro de las preguntas y finalidades con el nivel micro del análisis de las prácticas educativas reales. Identificar soportes y obstáculos del quehacer educativo para lograr el DS supone, en efecto, que no solo se clarifiquen los fines deseados, sino, ante todo, los valores que los fundamentan.

\section{El concepto de responsabilidad desde} la perspectiva del quehacer educativo

Hans Jonas repensó el marco y los fundamentos de una ética de la responsabilidad, que tiene en cuenta a las generaciones futuras y al mundo, tras las amenazas de la técnica moderna: "Actúa de tal modo que los efectos de tu acción sean compatibles con la permanencia de una vida humana auténtica" (1990). El problema, como lo plantea Siqueira es que "para que haya responsabilidad, es preciso que exista un sujeto consciente. Lo que ocurre es que el imperativo tecnológico elimina la conciencia, elimina al sujeto, elimina la libertad en provecho de un determinismo" (2009, p. 173). Si la noción de un DS lleva a pensar en un desarrollo que considere las cuestiones ambientales, sociales, económicas e intergeneracionales, entonces, la EDS implica una visión que no puede reducirse a la dimensión ambiental. ¿Cómo considerar una educación que favorezca el desarrollo del principio de responsabilidad? Creemos encontrar una clave en la noción de estudiantes autores, propia de las corrientes de la escuela nueva y, sobre todo, de Célestin Freinet, quien, ya en 1940, escribió con total claridad y audacia:

El esfuerzo pedagógico que realizamos supone cierta concepción social del devenir humano: es nuestra concepción republicana y democrática que espera de la escuela... que forme personas susceptibles de pensar libremente, y de actuar armoniosamente, al interior de la comunidad, capaces de defender las libertades conquistadas, sabiendo obedecer, pero competentes también para participar inteligentemente en la cuestión pública. (1940, p. 112)

Nicolas Go (2009) intenta teorizar la clave de esta transformación de las relaciones con el saber en la pedagogía de Freinet; ${ }^{6}$ muestra que el prin-

6 La misión principal de la escuela, para Freinet (1984), es ayudar a desarrollar las potencialidades de cada individuo. Es una escuela viva, una continuación de la vida del pueblo y del contexto, con sus dificultades y realidades. Para ello, propone un proceso educativo centrado en: a) el aprendiz: "toda pedagogía que no parte del educando es un fracaso, para él y para sus necesidades y sus aplicaciones más íntimas"; b) la labor del profesor: "poner a su disposición las técnicas más apropiadas y los instrumentos adecuados a estas técnicas". Es una educación para la acción que él denomina educación con el trabajo. Por eso, el conocimiento es acción, experiencia, práctica; la base de esta nueva pedagogía debe ser crear un ambiente de aprendizaje adecuado para que el aprendiz logre desarrollar actividades productivas y formativas. 
cipio fundamental que atraviesa su obra reside en la educación del deseo, entendido como facultad motriz de los aprendizajes:

Es posible lamentarse y quejarse, lanzar imprecaciones contra los niños de hoy que no saben ya ni escuchar, ni obedecer, que ya no respetan ni temen... La letanía es larga pero ahí están los hechos [...]. Podemos intentar la modernización de los instrumentos escolares, el mejoramiento de sus técnicas, o cambiar progresivamente las relaciones entre la Escuela y la Vida, entre los niños y los maestros, para adaptar y readaptar la escuela al medio, para un mejor rendimiento de nuestros esfuerzos comunes. (2009, pp. 3-4)

Constituir esta potencialidad creativa y escuchar a los estudiantes, "readaptando la escuela con la vida", permite entonces formarlos como autores de su propio desarrollo. Para comprenderlo mejor, conviene retomar la precisión que hacen Jacques Ardoino y Jean Guglielmi (1996), para quienes devenir autor no se reduce a ser actor, porque ser autor es desempeñar un rol en un escenario que es el suyo propio, mientras que el actor lo desempeña en un escenario cualquiera.

¿Qué dispositivo didáctico y pedagógico favorece tal "devolución radicalizada", como la llama Go (2009), en la cual el estudiante "vive como autor de sus tareas, autor de sus procesos de aprendizaje y co-autor del medio mismo, como proceso cooperativo"?7 Entre muchos dispositivos pedagógicos está el llamado "proyecto pedagógico de aula”. ${ }^{8}$ Como lo señala J-P. Boutinet (1990), la noción de proyecto surge en el Renacimiento, con el plan arquitectónico de Brunelleschi en Florencia. La complejidad, ligada a la diversidad de materiales, el número creciente de oficios así como los nuevos modos de construcción, fue dando lugar a una planeación metódica del traba- jo de concepción y creación; de modo que los dos momentos del proyecto (el de la concepción y el de la ejecución) se relacionan sin confundirse. Si este proceso racionalizante organiza el conjunto para alcanzar el objetivo buscado limitando, al mismo tiempo, los ensayos y errores empíricos, es claro que el proyecto no se reduce a su dimensión de anticipación. En efecto, esencialmente, el proyecto solo es exitoso sobre la base del análisis crítico de lo que ya existe (el contexto real) y se desarrolla desde la mirada de quien actúa. Como lo expresa J-B. Paturet (2002), el proyecto solo adquiere sentido en un futuro siempre abierto. Sartre había radicalmente definido el ser humano como proyecto de ruptura frente a las fatalidades: "La persona no es nada diferente a su proyecto, no existe sino en la medida en que se realiza..." (1970, p. 22).

Ahora bien, en la educación tradicional, hay que considerar, entre muchas otras cosas, dos fenómenos habituales, pero alarmantes:

- El hecho de que los estudiantes normalmente no eligen las acciones que deben realizar. Si bien no todos se quejan, muchos manifiestan su desacuerdo;

- Los proyectos educativos en los que participan no siempre permiten que los estudiantes elijan y tengan parte en las decisiones tomadas.

Al relacionar esto con lo que se dijo sobre la noción de proyecto, se puede pensar que si la participación de los estudiantes, tanto en el decidir qué acciones ejecutar como en la ejecución de las mismas, no es suficientemente perceptible, entonces el dispositivo pedagógico actúa como un freno del aprendizaje. A la inversa, los estudiantes que previamente participan en las decisiones so-

7 El aprendizaje colaborativo es un proceso social en el que, a partir del trabajo conjunto y el establecimiento de metas comunes, se genera una construcción de conocimientos; de acuerdo con Guitert y Giménez (2000), se da una reciprocidad entre un conjunto de individuos que saben diferenciar y contrastar sus puntos de vista, de tal manera que llegan a forjar un proceso de construcción de conocimiento.

8 El proyecto pedagógico es una estrategia de aprendizaje que articula teoría-práctica-investigación durante el proceso formativo de los estudiantes. 
bre lo que se pretende hacer se muestran responsables y motivados para colaborar en el proyecto. Se corrobora la importancia dada a sus decisiones y actos en expresiones como: "Nos dejaron elegir nuestro proyecto", "Es bueno para nosotros porque uno elige lo que uno hace, nos dan confianza". Sensibilizar e implicar a los estudiantes en las decisiones son elementos clave para una educación que promueva la capacidad de autonomía, en el marco del principio de responsabilidad, hace que los estudiantes se conviertan en autores de su propio desarrollo y de los conocimientos que adquieren.

\section{El concepto de democracia al interior de prácticas educativas innovadoras}

Esta idea (la educación reside esencialmente en el principio de la responsabilidad compartida) lleva al concepto de que todo individuo es parte integrante de una comunidad más amplia - la humanidad-, en la cual es llamado a participar responsablemente. La cuestión de la democracia aparece aquí claramente; democracia, en el sentido de Dewey (1995), cuando la definió como el lugar donde el conjunto de los ciudadanos participa en la gestión y el control de la vida de la comunidad. La escuela, desde entonces, no puede reducirse a adaptar al niño a la sociedad de los adultos (socialización), sino que se anticipa para hacer vivir hoy las cuestiones de una acción colectiva necesaria para el mañana (emancipación). Como lo explica el autor:

Tan evidente es, en efecto, la necesidad de enseñar y aprender para la existencia continuada de una sociedad, que puede parecer que estamos insistiendo indebidamente sobre un lugar común. Pero esto tiene su justificación en el hecho de que tal insistencia es un medio de evitar que caigamos en una noción escolástica y formal de la educación. (1995, p. 15)

Por eso, Dewey reivindica la "necesidad de modos de enseñanza más fundamentales y dura- bles" con la condición de considerar su relación con las nociones de comunidad y de comunicación, que permiten la participación de cada uno en una obra común. Esta participación no se entiende en términos de entrenamiento, sino, más bien, como elaboración de posibilidades de actuar, de reaccionar y de aprehender ante un problema cuando este se plantea; dicho de otro modo, no como una respuesta única y fijada para siempre, sino como las posibilidades de las que cada uno dispone en un proceso educativo. Esta distinción entre resultado y proceso se encuentra igualmente en Dewey (1995, pp. 53-54).

En Francia, Célestin Freinet planteó un concepto de educación que iba en el mismo sentido, como una actividad de desarrollo de los estudiantes para que ellos aprendan juntos a partir de las experiencias realizadas. En su obra, Las técnicas Freinet de la escuela moderna (1999), el autor explica las invariantes pedagógicas que se requieren para innovar las prácticas de enseñanza. La invariante número 27 precisa: "se prepara la democracia del mañana mediante la democracia hoy en la escuela", y tal preparación supone varios presupuestos: uno, explicar qué significa "democracia en la escuela”; otro, clarificar los roles más pertinentes del maestro y del estudiante para un ejercicio democrático; tercero, una concepción e implementación de situaciones de clase, adecuadas para ejercer la democracia, sin caer en la superficialidad o el formalismo. Una educación adecuada reúne esas preocupaciones fundamentales porque la expresión "educación para" compromete ciertos dispositivos para actuar, que no se reducen a la repetición de gestos o eslóganes.

En 1988, la Unesco inscribe la "educación para el desarrollo" al interior del Proyecto Educativo Planetario, considerando que constituye la finalidad misma del desarrollo humano. Esto invita a considerar una dimensión, específicamente contemporánea, de la educación que no puede seguir centrada en los ideales y valores de la Modernidad. En efecto sí, como lo explica Lucie Sauvé (1999), la Modernidad se caracteriza por la creencia en el progreso científico y tecnológico, y su epistemología se fundamenta en los valores de la ob- 
jetividad y la racionalidad instrumental; desde el punto de vista ético, los valores de la Modernidad son antropocéntricos y la libertad del individuo solo termina en las fronteras del respeto de la libertad del otro. Con el liberalismo salvaje y los regímenes totalitarios, estas esperanzas modernas se desmoronaron. La Posmodernidad debe ahora hacer frente no solo a la deconstrucción y a la complejidad, sino también a una cierta radicalización y dramatización frente al futuro. La educación posmoderna, lejos de justificar a priori las elecciones mediante concepciones teóricas ya establecidas, prefiere el proceso de discusión crítica sobre los problemas encontrados. Sin entrar en la polémica de aquellos que, como Lucie Sauvé, afirman que la expresión "desarrollo sostenible" comporta en su "corazón” ecos de la Modernidad y de la creencia en el progreso, aparece claramente que el modelo de la pedagogía posmoderna supone siempre una participación responsable en las deliberaciones previas a la toma de decisiones. Según Jean-Pierre Pourtois y Huguette Desmets, el modelo pedagógico posmoderno será

[...] un nuevo sistema complejo que tiene en cuenta las dimensiones afectivas, cognitivas, sociales y éticas del individuo así como los conflictos inherentes a las cuestiones culturales y a sus negociaciones [...] Los aprendientes deberán ser considerados como sujetos a reconocer y a entender, sin condescendencia ni facilidad. (2002, p. 43)

Restituir su lugar al sujeto y, sobre todo, su responsabilidad ética; trabajar con una metodología racional, con un fondo de incertidumbre fundamental; esos serían los elementos característicos de tal pedagogía. La dificultad principal para la escuela es responder a esas demandas sin dejarse instrumentalizar por una política partidista que la apartaría de sus propias finalidades.

En este enfoque posmoderno, varios pedagogos han formulado conceptos como: organización aprendiente, comunidad de aprendizaje y comunidad de investigación, incluso comunidad de práctica e investigación. A la luz de esto que se acaba de precisar, habrá que examinar más a fondo el sentido y clarificar sus campos de atribución para captar los apoyos y obstáculos que plantean para una EDS. Aquí se formula la hipótesis siguiente: educar para el DS requiere actitudes y procesos propios de la democracia participativa.

\section{Organización aprendiente y comunidad aprendiente}

El concepto de organización aprendiente proviene de los trabajos de Argyris y Schön (1978) y remite a la idea de que una organización es aprendiente desde el momento en que sus miembros construyen aprendizajes, individuales y colectivos, sobre la base de acciones (prácticas) realizadas, que les permiten confrontar los desafíos que se le presentan a la organización. En psicosociología de las organizaciones, la noción de organización tiene que ver tanto con una empresa como con un establecimiento, para los cuales la meta consiste en lograr los objetivos a pesar de los desafíos que se le presentan. Inicialmente, el término no fue concebido en relación con la escuela.

Léopold Paquay explica que la escuela no es, de entrada, calificada de organización aprendiente porque su objetivo reside, ante todo, en el aprendizaje de los estudiantes, no en el éxito del establecimiento mismo. Pero se puede comprender que invertir los objetivos es factible: en un caso, el aprendizaje de los estudiantes incide en el aprendizaje del establecimiento, pero los estudiantes mismos solo son considerados como medios; en otro caso, al contrario, el aprendizaje de los estudiantes es el objetivo al que se dirige el conjunto de los actores. Es por eso que Paquay considera que la escuela solo puede ser calificada de organización aprendiente "cuando la dinámica de ejecución de sus objetivos es asegurada al máximo por un personal comprometido en una dinámica de proyecto y de aprendizaje que le permite renovarse continuamente para responder a los desafíos de los cambios" (2005, p. 58).

Los expertos de la Organización para la Cooperación y el Desarrollo Económicos (OCDE) (2003) incitan a las escuelas a llegar a ser organizaciones aprendientes y a los maestros a 
convertirse en artesanos de proyectos colectivos, continuando con su desarrollo profesional a lo largo de la vida. Así, si las escuelas son llamadas a convertirse en organizaciones aprendientes, los maestros son llamados a constituirse en comunidad profesional de aprendizaje. Como lo expresó Philippe Perrenoud (1997), cambiamos de paradigma escolar, pasando de una escuela que quiere transmitir conocimientos a una escuela que quiere generar competencias y actitudes ciudadanas. Este cambio de paradigma requiere de nuevas competencias en los maestros; de ahí la importancia dada a la capacidad de análisis de su propia práctica profesional como a aquella de formarse permanentemente; pero requiere, igualmente, de nuevas competencias para los estudiantes. Por eso no es cuestión de azar que los referenciales de competencias se multiplican en numerosos campos disciplinares. Las competencias esperadas, para los estudiantes y maestros, tienen siempre que ver con su autonomía. Y, por definición, esta solo puede construirse sobre la base de un proceso activo y personal. La autonomía no se decreta; ella se adquiere ejerciéndola.

Monica Gather-Thürler (2000), especialista en cuestiones de innovación en equipos de maestros, identifica las características requeridas para que estos equipos funcionen como comunidades aprendientes, como organismos vivos con disposición para aprender, con apertura a la innovación; entre ellas, la competencia para debatir sobre valores y preguntas; la de tomar conciencia de las cuestiones sociales en las prácticas cotidianas; aquella de desarrollar un sentimiento de poder sobre la evolución de las cosas. Estas tres competencias corresponden al difícil y complejo campo de la EDS. Así, desde el punto de vista de un proyecto pedagógico, el equipo docente funciona como una comunidad aprendiente, usa la competencia reflexiva, entendida como el retorno del pensamiento sobre sí mismo.

Como lo explicó Richard Wittorski (2001), la reflexividad supone que el individuo puede separarse de su marco de referencia habitual y recurrir a otros elementos de lectura de la actividad. Retomando las características de Gather-Thürler, es fácil que los equipos docentes desarrollen un sentimiento de poder sobre la evolución de las cosas; al contrario, no pasa lo mismo para tomar conciencia de lo social en las prácticas cotidianas, pues tienden a juzgar a los estudiantes como quienes tienen dificultad para tomar decisiones, no saben buscar entre varias soluciones posibles (y es claro que eso no tiene que ver tanto con las prácticas escolares cuanto con una debilidad inherente).

Finalmente, respecto a la competencia para debatir sobre valores y preguntas, no es muy frecuente en la educación tradicional. Esta competencia es, sin duda, la más exigente en la medida en que los valores y las preguntas son los menos perceptibles en la práctica. Frecuentemente, en efecto, hablar sobre las intenciones del hacer difiere de lo que deja ver el hacer mismo. Integrar al marco de la EDS el concepto de organización aprendiente hace posible que la escuela se organice para construir nuevas prácticas y afrontar así la permanente renovación de los desafíos que se le plantean. Considerar la escuela como una organización, capaz de aprender de sí misma y por sí misma, en medio de la complejidad de sus aspiraciones y contextos, lleva a otorgarle cierto margen de autonomía, más allá de sus cuestiones vitales y sociales. Ahora bien, tener en cuenta todo esto, ¿no implica tener en cuenta el concepto de comunidad aprendiente que, según Gutiérrez y Stone, pretende "organizar el intercambio de palabras, la expresión del respeto mutuo y la demostración de una escucha activa" (1997, p. 33)?

Si se quiere precisar la pertinencia de recurrir al concepto de comunidad aprendiente, conviene apoyarse en los trabajos de Jean-Claude Kalubi (2005), quien considera que, dado que el aprendiente es el principal autor e iniciador de sus logros, es preferible favorecer aquellos procesos que privilegian la construcción de acciones por los aprendientes mismos. Además, si el aprendizaje se comprende como un proceso social centrado en valorar las interacciones, hay que privilegiar los contextos y los marcos organizacionales, favoreciendo dichas interacciones. 
El interés mayor del concepto de comunidad aprendiente reside en que suscita una reflexión sobre el lugar ocupado por cada participante y el reconocimiento de los esfuerzos mutuos de los diversos actores. En el caso de la EDS, podríamos decir que pensar la escuela y la clase como una comunidad aprendiente, integrando las familias, los pares, el barrio, las instancias municipales [...], invita a tenerlas en cuenta en el proyecto inicial, así como también a favorecer los contactos, los intercambios y las decisiones tomadas en común. Por eso, la comunidad aprendiente es una comunidad que aprende de unos, de otros y de experiencias comunes. Tal aprendizaje no podría efectuarse sin la voluntad inicial de aceptar considerar al otro como compañero en la discusión y la decisión, y también de aprender de él, sea quien sea: niño, novicio, experto, sabio, ignorante... Ahora bien, para todo maestro, esta es una reflexión difícil de considerar. ¿Cómo pensar la asimétrica relación alumno/maestro en los planes de estudio y en los reglamentos, reconociendo una igualdad fundamental con el otro? ¿Cómo cambiar de postura sin perder el estatuto de adulto y de profesional como referente para los estudiantes? Igualmente, ¿cómo considerar y establecer los contactos con los diferentes actores de un proyecto - las familias, los habitantes del barrio, los representantes municipales?

Esas cuestiones, de una manera u otra, se plantean al maestro. Más allá de diferencias a nivel de las preguntas y de la organización, parecería que esta sea la lógica misma de la noción de proyecto. Esta noción de "lógica de acción" se refiere a los trabajos de Bernoux, para quien

[...] una lógica de acción nace de los sentidos que el individuo da a la acción que emprende, dependiendo de la situación de la misma. Ella tiene sus raíces en las representaciones, las imágenes activas que los individuos se han forjado en el curso de su vida, imágenes vinculadas a sus identidades. Ella permite explicar el tipo de razonamiento emprendido. (2004, p. 259)

¿Esta alteridad de la lógica de acción significa una ruptura que hace imposible la comunicación?
La diferencia percibida por los maestros, entre las dos lógicas, reside esencialmente en el nivel de la temporalidad y de la eficacia en el avance del proyecto. En el proyecto pedagógico de aula, el proceso ocurre tanteando; la programación no puede organizarse totalmente, ni preverse en sus limitaciones y contenidos.

En el proyecto educativo "institucional", los objetivos parecen estar establecidos de una vez para siempre; es la lógica programática que no deja lugar al debate sobre el objetivo y los medios para lograrlo. La eficacia organizacional parece aquí lo primero. Se requiere una toma de conciencia de que si, para una escuela o un grupo de estudiantes, convertirse en socio de un proyecto ampliado - comunal, municipal o de grupopuede parecer un apoyo favorable al impacto social del proyecto, este mismo apoyo puede transformarse en freno, incluso en obstáculo, si las partes no se explican claramente sobre los sentidos y problemas de su participación.

Aquí hay una relación con los trabajos de Bernoux (2004) a propósito de las búsquedas colaborativas entre investigadores y practicantes o profesionales. En ellos, en efecto, un contrato de investigación debe definir una doble pertinencia, tanto para los investigadores como para los practicantes o profesionales, lo que permite clarificar las posiciones y expectativas de cada uno de los socios. Por otra parte, lo que explica las diferentes lógicas de acción, reside probablemente en lo que Crozier y Friedberg llaman la parte de incertidumbre: "Si hay una incertidumbre, los actores capaces de controlarla la usarán en sus transacciones con aquellos que dependen de ella. Porque ¿lo que es incertidumbre desde el punto de vista de los problemas es poder desde el punto de vista de los actores?” (1977, pp. 265-313). Así, la acción colectiva procedente de las políticas públicas tiende a reducir los márgenes de incertidumbre sin duda para limitar los riesgos de responsabilidad en las acciones ejecutadas. Estamos lejos, en este caso, de los conceptos de organización o de comunidad aprendiente porque se trata menos de aprender juntos que de hacer, sin preocuparse de la participación real de los interesados. 


\section{Comunidad de investigación y comunidad de práctica}

El concepto de comunidad de investigación, particularmente reivindicado por M. Lipman (2006), se inscribe como una prolongación del pensamiento pragmatista de C. S. Peirce (1931) y de Dewey. Para Peirce, inventor del concepto, la comunidad de investigación permite a los investigadores confrontar sus diferentes puntos de vista y, por sí misma, justificarlos para llegar a determinar el más pertinente. Discutir en común a fin de poder juzgar la calidad de una aserción permite comprender los valores subyacentes dados a la intersubjetividad y al constructivismo. Es, en efecto, reconocido el carácter "social y construido del saber científico que remplaza la verdad por la viabilidad" (Daniel, 2005, p. 25).

Discutir o dialogar, para Lipman y los pragmatistas, no se reduce simplemente a intercambiar; se trata más bien de participar juntos en la verificación de la viabilidad de los discursos en juego. Como lo precisa igualmente Daniel (2005), hay una comunidad de investigación cuando personas reunidas intercambian entre ellas, de modo auténtico, con el objetivo de desarrollar una reflexión crítica y responsable. Es clara la relación con el actuar comunicativo de Habermas (1987). El saber procede de la reflexión crítica entre pares. Pero, precisamente, ¿qué son los pares en el marco escolar y cómo transponer este concepto a la clase? Marie Agostini (2007) recuerda varios elementos clave: por una parte, que la maduración del razonamiento del niño tiene que ver con un proceso no espontáneo, sino dinámico en el sentido de que el razonamiento se desarrolla en función del ambiente que lo interpela; por otra parte, que la discusión y el ejercicio del juicio le permiten al pensamiento afirmarse, por ensayo y error, como lo muestran dos figuras remarcables de la filosofía: Sócrates y Montaigne.

Si Peirce entendía la noción de comunidad como los mismos investigadores; Lipman entiende la clase a condición de que los estudiantes elaboren sus ideas, sobre el fondo de razonamientos, asumiendo la responsabilidad de participar en la investigación común, lo cual requiere poder revisar su propio juicio, y escuchar buscando comprender el punto de vista del otro. La búsqueda personal de sentido, a partir de los intercambios, remite aquí igualmente al concepto de Dewey (1995), para quien el conocimiento consiste, ante todo, en la adquisición de dispositivos para comprender y actuar. Ahora bien, precisamente, adquirir estos dispositivos es una de las preocupaciones de la EDS, porque no se trata tanto de proporcionar respuestas de una vez para siempre como de aceptar ser interpelado, en todo momento, sobre esas cuestiones para reajustar, según vaya siendo necesario, las primeras decisiones tomadas. Como lo definió Lipman (1993), la reflexión crítica es un modo de reflexión autocorrectiva, permeable al contexto, y apoyada sobre criterios con miras a emitir un juicio. Se comprende que esta reflexión equivale a ser capaz de problematizar en comunidad. Para hacerlo, ¿cuáles serían los soportes? El autor señala la lista de las aptitudes y disposiciones requeridas:

- Aptitud para los razonamientos, sean de tipo inductivo, deductivo o analógico;

- Aptitud para la investigación, entendida en términos de capacidad de observación, descripción, narración;

- Aptitud para la conceptualización, comprendida como capacidad de definir, de clasificar;

- Aptitud para la formulación, que supone la escucha, la comprehensión, la escritura;

- Dispositivos críticos que lleven a la curiosidad, la búsqueda de explicación, el juicio, el comprender la noción de criterización y la aceptación del cuestionamiento.

Por otra parte, la cuestión fundamental permanece: ¿esas disposiciones son naturales o pueden ser adquiridas mediante prácticas realizadas en el marco de un proyecto de EDS? Pensamos que esos indicadores de aptitud sirven para identificar lo que sería importante lograr con un dispositivo pedagógico determinado. Dicho de otra 
forma, esos criterios proporcionan hipótesis para impulsar estrategias de acción. Se apoyan sobre una concepción del saber y del rol de la escuela: la concepción socioconstructivista del aprendizaje donde el saber resulta de un proceso abierto de coconstrucción, relativo a las normas de momento admitidas según los criterios establecidos por la sociedad, el grupo de referencia, la cultura.

Asimismo, precisar la importancia del contexto subraya la relación entre la elaboración del saber y el contexto experiencial de su producción. Estas concepciones subyacentes necesitan ser explicitadas para aprehender claramente las cuestiones y las finalidades esperadas, so pena de formar en el espíritu de los actores una mezcolanza de representaciones propias, a veces confusas y, en el peor de los casos, contradictorias e improductivas. Tales confusiones, aplicadas a la EDS, constituirían un obstáculo real para su dimensión específicamente praxeológica; impedirían la ejecución de proyectos in situ que busquen un bienestar para todos. A nivel de la EDS, los proyectos pretenden un bienestar para todos, incluso si ellos solo se concretan a nivel local. Considerar esta doble relación de lo local y lo colectivo o global, a escalas diferenciales, requiere de una plasticidad y una creatividad hasta ahora ausentes en los contenidos trabajados tradicionalmente en la escuela.

Es por eso que el objetivo de la EDS consiste, para la escuela, en constituirse no solamente como comunidad de investigación, sino también como comunidad de práctica, inspirada en los planteamientos de Wenger (2005), es decir, suponiendo un compromiso mutuo de todos sus miembros, acciones colectivas y la constitución de un repertorio compartido de recursos. No obstante, a fin de no correr el riesgo de encerrar a las escuelas en sí mismas, esta comunidad debe entenderse como Escuela, en el sentido amplio, que se esfuerza por mutualizar sus prácticas; lo que supone hacerlas leíbles y explícitas, dando su lugar a los estudiantes, a sus familias, a la noción del otro en general y al mundo. Ahora bien, la idea misma de comunidad debe ser pensada; no se trata de grupo, equipo o de red, en tanto que la pertenencia a una comunidad implica un compromiso mutuo de los participantes, lo que favorece la coherencia comunitaria. Es que la noción de comunidad es, sin duda, una de las más difíciles de percibir. En realidad, lejos de pensar que la EDS está llena de obstáculos, obliga a los participantes a no descuidar esas dimensiones comunitarias porque les son inherentes.

\section{A modo de síntesis conclusiva}

La dimensión posmoderna, en lo que concierne a las cuestiones educativas, supone aceptar dos criterios: la complejidad, por una parte, y la contextualidad de los objetos del saber, por otra parte. Es necesario el diálogo entre los diversos tipos de saberes y de enfoques a fin de poder aprehender. El proceso de discusión crítica se privilegia para fundamentar una toma de posición ética, responsable y democrática, no solo en cuanto a los objetos de saber, sino también en cuanto a las decisiones mismas, porque ellas comprometen a toda la comunidad humana en su relación con el mundo aquí, ahora y por venir.

En el marco de la EDS, el lugar dado a los objetos del saber y a la discusión razonada entre todos, con miras a participar en la construcción de una decisión razonable y viable para la humanidad y el planeta, no solamente in situ, sino igualmente a una escala de tiempo y de espacio a más amplio término, se vuelve desde entonces determinante. Siguiendo a John Dewey, que pensaba que si el sistema educativo había fracasado era porque se había pedido a los estudiantes aprender resultados y soluciones hechas, más que estudiar problemas y pedirles que ellos mismos llegaran a ser investigadores, es claro que la complejidad de la situación actual y los riesgos que comporta para la perpetuación misma de la vida hacen pensar que es necesario, como lo formuló Lipman (2006), "aprender a actuar de manera razonable" sobre la base del siguiente proceso: 
- Expresar el sentimiento de que existe un problema;

- Formular el problema;

- Enunciar un objetivo deseable;

- Identificar medios posibles;

- Prever las consecuencias;

- Elegir una solución;

- Elaborar un plan;

- Evaluar los efectos.

Sin olvidar las dimensiones sensibles y afectivas que entran en la elaboración de los juicios. Si saber sin discutir es imposible, y discutir sin saber es vano, entonces decidir sin saber ni discutir juntos no tiene ninguna validez porque la decisión compromete el futuro de todos y para todos.

Es por eso que, de facto, la EDS supone una ética. Como lo precisa Hans Jonas, es "el futuro indeterminado, más que el espacio actual de la acción, lo que forma el horizonte significativo de la responsabilidad" (1990, p. 10). La noción misma de durabilidad tiene este precio. El aporte de la ética se sitúa, esencialmente, en su competencia para interrogar y buscar cómo apreciar el valor y la pertinencia de los medios y las elecciones posibles que superan el interés particular. Ahora bien, el interés de la comunidad humana trans-generacional compromete el ejercicio de un juicio de tipo reflexivo, que no conoce conceptos a priori a partir de los cuales se pueda deducir lógicamente un contenido predeterminado.

Por eso hay que pensar que la EDS supone no solamente objetos de saber que implican prácticas de juicios determinantes y preocupaciones éticas, teleológicas, que necesitan de juicios reflexivos; sino, igualmente, experiencias de proyectos reales que permitan a la comunidad, formada por los estudiantes y sus familias, así como por los maestros y otros integrantes de la comunidad, dar sentido y valor al actuar razonable que inscribe su acción en un bien común lo más amplio posible. Por eso mismo se comprende que a la comunidad no le basta que el grupo tenga líderes, sino más bien requiere de autores, como lo planteó Freinet.

Este actuar razonable no puede darse sin creatividad porque siempre está confrontado obstáculos inesperados y complejos. La plasticidad y la flexibilidad son, entonces, requerimientos no solo para encontrar otros modos de actuar, sino también para dotarse de nuevas normas. En eso consiste el carácter esencialmente creativo del actuar humano, tal como Hans Joas (2013) $)^{9}$ lo precisó al cuestionar la teoría de la diferenciación, el paradigma dominante en la explicación de los cambios sociales, contraponiendo su propia teoría de la activación de la responsabilidad colectiva para la gestión de los asuntos comunes.

Esa es la propuesta para una democracia creativa en el marco de una EDS que, en la perspectiva del PEI de Uniminuto adquiere las siguientes connotaciones:

En dicha perspectiva, para UNIMINUTO el conocimiento es profundamente reflexivo y tiene que ver también con el conocimiento de sí mismo y del otro, con el sentido pleno de una vida llevada con propósito. Del compromiso que se tiene con el otro, con el entorno, con la vida. El conocimiento es ante todo un acto interactivo, lo que se asocia a la importancia de la concordia para la comunicación, para la vida en comunidad, para las prácticas sociales recurrentes que devienen en cultura, en una manera de vivir juntos. Por eso UNIMINUTO, como institución al-

9 Así como el neofuncionalismo, encarnado en la obra de Luhmann, intentó renovar el paradigma funcionalista, Joas propone, en total confrontación con el neofuncionalismo y con el paradigma de la acción racional, otro alternativo e innovador que replantea la teoría de la acción partiendo de reconocer el carácter creativo del actuar humano, puesto ya de relieve por G. H. Mead y John Dewey. 
ternativa de educación superior, se compromete con el diálogo de saberes y el reconocimiento de otras formas de producir y generar conocimiento pertinente para la sociedad. Temas como la educación para el desarrollo y la innovación se constituyen en preocupaciones básicas. Los saberes de las comunidades ancestrales, así como los desarrollados por las comunidades agrarias, campesinas, de pescadores o de los habitantes urbanos, se aúnan a los saberes científicos y disciplinares, en ese proceso praxeológico de reflexión desde la acción y la práctica que impregna el quehacer educativo institucional. El desarrollo de innovaciones sociales que permitan sortear pertinentemente las diferencias y el conflicto con base en el diálogo, la Noviolencia, el reconocimiento mutuo y la empatía, así como la construcción de ciudadanía y de nación, son centrales para el cumplimiento de su misión educativa. (Uniminuto, 2014, cap. IV, pp. 34-35)

\section{Referencias}

Agostini, M. (2007). La philosophie dès l'école maternelle. Actes du colloque de l'Actualité de la Recherche en Education et Formation. Strasbourg.

Ardoino, J. y Guglielmi, J. (1996). Prefacio. En A. Christian Alin, Etre formateur quand dire c'est écouter. Paris: L'Harmattan.

Argyris, C. y Schön, D. (1978). Organizational learning: A theory of action perspective. Reading, MA.

Bachelard, G. (2000). La formación del espiritu cientifico: contribución a un psicoanálisis del conocimiento objetivo (23 ed.). México: Siglo XXI.

Bernoux, P. (2004). Sociologie du changement dans les organisations. Paris: Seuil.

Boumard, P. (1996). Célestin Freinet. Paris: PUF.

Boutinet, J.-P. (1990). Anthropologie du projet. Paris: PUF.

Daniel, M.-F. (2005). Présupposés philosophiques et pédagogiques de Matthew Lipman et leurs applications. En L. Claudine, La philosophie pour enfants. Bruxelles: De Boeck, pp. 25-46.

Dewey, J. (1995). Democracia y educación. Madrid: Morata.
Crozier, M. y Friedberg, E. (1977). L'acteur et le système, Seuil. En BERNOUX, P. A quoi sert la sociologie des organisations?. Sciences Humaines, Hors (20), p. 12-15.

Freinet, C. (1999). Técnicas Freinet de la escuela moderna. México: Siglo XXI.

Freinet, C. (1984). Por una escuela del pueblo. México: Fontanara.

Gather-Thürler, M. (2000). Innover au coeur de l'établissement scolaire. Paris: ESF.

Go, N. (2009). Une approche complexe des apprentissages. La primauté du processus en pédagogie Freinet. Penser l'Education, (25), 19-48.

Guitert, M. y Giménez, F. (2000). El trabajo cooperativo en entornos virtuales de aprendizaje. En J. Duart y A. Sangra (eds.). Aprender en la virtualidad. Barcelona: Gedisa, pp. 113-134.

Gutiérrez, K. y Stone, L. (1997). Creating communities of effective practice: Building literacy for language minority children. En: J. Oaks \& K. Quartz (Eds.), 94th NSSE Yearbook: Creating New Educationnal Communities. Chicago: University of Chicago Press, pp. 32-52.

Habermas, J. (1987). Teoría de la acción comunicativa. Taurus: Madrid. 
Joas, H. (2013). La creatividad de la acción. Madrid: Centro de Investigaciones Sociológicas.

Jonas, H. (1990). Le principe responsabilité, une éthique pour la civilisation technologique (trad. Française J. Greisch). Paris: Flammarion.

Kalubi, J. C. (2005). Trois questions sur le développement des communautés d'apprentissage en milieu scolaire. En B. Mabilon-Bonfils (dir.), Violence scolaire et cultures. Paris: L'Harmattan, pp. 161-172.

Lipman, M. (1993). Renforcer le raisonnement et le jugement par la philosophie. En C. Leleux (2005), La philosophie pour enfants. Le modèle de Matthew Lipman en discussions. Bruxelles: De Boeck, pp. 11-24.

Lipman, M. (2006). A l'école de la pensée. Bruxelles: De Boeck.

Paquay, L. (2005). Devenir des enseignants et formateurs professionnels dans une "organisation apprenante"? De l'utopie à la réalité. Recuperado el 17 de enero de 2015 de http:// www.tandfonline.com/doi/abs/10.1080/0261 9760500093099\#preview

Paturet, J. B. (2002). Le projet comme «fiction commune». Empan, (45), 63-68.

Peirce, C. S. (1931). Collected papers. Cambridge MA: Press of Harvard University.

Perrenoud, P. (1997). Construire des compétences à l'école. Paris: ESF.
Pourtois, J. P. y Desmets, H. (2002). L'éducation postmoderne. Paris: PUF.

Sartre, J. P. (1946/1970). L'existentialisme est un humanisme. Paris: Nagel.

Sauvé, L. (1999). Environmental education, between modernity and post modernity. Searching for an integrative framework. Canadian Journal of Environmental Education, 4, 9-35.

Siqueira, J. de (2009). El principio responsabilidad de Hans Jonas. Revista BioEthikos, 3(2), 171-193. Recuperado el 18 de enero de 2015 de www.saocamilo-sp.br/pdf/bioethikos/71/171-193.pdf

Uniminuto. (2014). Proyecto Educativo Institucional (PEI). Bogotá: autor.

Useche, O. (2012). Educando para hallar alternativas al desarrollo. En L. López y A. Orrego (eds.), Deconstruyendo la educación para el desarrollo: una mirada desde Latinoamérica. Bogotá: Uniminuto.

Wenger, E. (2005). La théorie des communautés de pratique, apprentissage, sens et identité. Canada: Les Presses de l'Université de Laval.

Wittorski, R. (2001). Contribution de l'apprentissage expérientiel et de l'action à la pratique professionnelle. En Mackiewiez. Praticien et chercheur. Paris: L'Harmattan, pp. 107-118. 